\title{
DESAJUSTE EDUCATIVO EN EL MERCADO LABORAL DE MÉXICO Y SU EFECTO EN LOS SALARIOS
}

\section{EDUCATIONAL MISMATCH IN THE MEXICAN LABOR MARKET AND ITS EFFECT ON WAGES}

\author{
Norma Aída Valenzuela Sánchez \\ UNIVERSIDAD AUTÓNOMA DE \\ NuEvo LEÓN \\ norma.valenzuelasn@uanl.edu.mx
}

Rosario Alonso Bajo UNIVERSIDAD AUTÓNOMA DE SINALOA alonsobajo@yahoo.com.mx
Jorge OMar Moreno Treviño

UnIVERSIDAd AutónOMA dE Nuevo LeÓN

jorge.morenotr@uanl.edu.mx

\section{RESUMEN}

Se examina el nivel y los efectos del desajuste educativo en el mercado laboral de México. En primer lugar se mide la magnitud de desajuste y en segundo se estima la ecuación salarial extendida de Duncan y Hoffman (1981) para obtener, por separado, los retornos a la educación requerida, el rendimiento de sobreeducación y la penalización a la subeducación. El modelo se prueba con datos de la Encuesta nacional sobre niveles de vida de los hogares (ENNViH). La escolaridad requerida para cada ocupación se deriva de obtener los niveles medios de escolaridad para cada puesto de trabajo. Los resultados muestran que la educación excedente o subutilizada se recompensa, pero a una tasa más baja que la educación requerida.

Palabras clave: desajuste educativo, sobreeducación, ocupación, capital humano, diferencias salariales 


\section{ABSTRACT}

This article examines the level and effects of educational mismatch in the labor market in Mexico. First, the magnitude of the mismatch is measured, and secondly, the extended wage equation of Duncan and Hoffman (1981) is estimated to obtain separately the returns to the required education, the performance of over education and, the penalty to the sub-education The model is tested with data from the Mexican Family Life Survey (MXFLS).

The schooling required for each occupation is derived from obtaining the average levels of schooling for each job. The results show that the surplus or underutilized education is rewarded but at a lower rate than the education required.

Keywords: educational mismatch, over education, occupation, human capital, salary differences.

Clasificación JEL: I21, J24, J31

\section{INTRODUCCIÓN}

La relación positiva entre educación e ingresos está bien documentada y sustentada, principalmente en la teoría del capital humano (Becker, 1993; Mincer, 1974). Desde este punto de vista, la educación conduce a mayores ganancias a través de su impacto en la productividad, el aumento de la educación aumenta la productividad de un individuo en el lugar de trabajo $\mathrm{y}$ el incremento de la productividad conduce a mayores ingresos.

No obstante, la expansión educativa experimentada en la mayoría de las economías en las últimas décadas ha originado la interrogante si el mercado laboral tiene la capacidad para proporcionar empleos altamente calificados, que utilice plenamente la inversión en educación individual y social. En un inicio este tema despertó el interés de los investigadores estadounidenses en los años setenta (Freeman, 1976), posteriormente 
se extendió a Europa (Chevalier y Lindley, 2009) y en la actualidad ya existen estudios al respecto para diversos países tanto desarrollados como en vías de desarrollo (Kucel, 2011; Leuven y Oosterbeek, 2011; Quinn y Rubb, 2006; Flisi et al, 2017).

Las investigaciones sobre el desajuste educativo se han centrado, principalmente, en la rentabilidad de un año extra de educación y sus consecuencias en términos de productividad y satisfacción laboral (Duncan y Hoffman, 1981; Sicherman, 1991; Capsada Munsech, 2017; McGuinness y Pouliakas, 2017). Los resultados muestran que una determinada proporción de la población ocupada presenta un desajuste entre la escolaridad poseída y la requerida por los empleos, ya sea por exceso de educación (sobreeducación) o por carencia (subeducación). Sin embargo, encuentran que la educación excedente no es completamente improductiva, tiene un efecto positivo y significativo en las tasas salariales. El coeficiente estimado en los años de educación excedente es aproximadamente la mitad del coeficiente en los años requeridos de educación.

La recompensa a la escolaridad varía entre los diferentes tipos de trabajadores y entre los diversos entornos de trabajo. Thurow (1975) desarrolla un modelo de competencia laboral en el que los productos marginales y, por lo tanto, las ganancias, se asocian con los trabajos no con los individuos. Como señala Sattinger (1993), la cantidad con la que un trabajador puede contribuir a la producción depende, típicamente, de qué trabajo realiza este. Ello ocurre porque los trabajos requieren muchas tareas diferentes, y las actuaciones humanas en esas tareas son extremadamente diversas; además, los sectores industriales utilizan tecnologías diferentes que dependen de distintas combinaciones de habilidades humanas.

El objetivo de esta investigación es medir la correspondencia existente entre el grado escolar de los trabajadores en México y el nivel de educación requerido por la ocupación donde laboran. En primer lugar se mide la magnitud de desajuste y en segundo se estima la ecuación salarial extendida de Duncan y Hoffman (1981) para obtener por separado de los retornos a la educación requerida, la sobreeducación y la subeducación. 


\section{2. ¿CÓMO DEFINIR Y MEDIR DESAJUSTE EDUCATIVO?}

El término desajuste educativo o desajuste profesional ha sido discutido en la literatura tanto en términos teóricos (Leuven y Oosterbeek, 2011; Kucel, 2011; McGuinness y Pouliakas, 2017; Capsada Munsech, 2017) como empíricos (Sicherman, 1991; Quinn y Rubb, 2006; Castillo, 2007; Flisi et al, 2017; Mehta et al, 2011). En general, todos los estudios coinciden con lo siguiente: El desajuste educativo se define como la diferencia entre el nivel de escolaridad alcanzado o completado de un trabajador y el nivel de escolaridad requerido para la ocupación donde labora.

La definición anterior, aunada a los tres conceptos utilizados originalmente por Duncan y Hoffman (1981) son los aceptados en esta investigación para medir el desajuste educativo.

I. Sobreeducado: se considera que un trabajador está sobreeducado cuando la educación que aporta al mercado laboral excede la requerida para su ocupación o trabajo. Por lo tanto, la sobreeducación es básicamente un desajuste entre la educación de un individuo y los requisitos educativos para realizar el trabajo.

II. Subeducado: se considera un trabajador subeducado cuando el nivel educativo es inferior al requerido por el puesto de trabajo.

III. Adecuadamente ubicado: cuando el nivel educativo corresponde con el requerido por su puesto de trabajo.

El estudio de desajuste educativo ha presentado importantes desafíos para los investigadores, principalmente en su medición, el primer paso es definir el nivel requerido de escolaridad en cada ocupación. Existen tres enfoques diferentes: a) Método de autoevaluación, b) Análisis normativo (o de trabajo) y c) Método estadístico. No está definido cuál de los tres métodos es el mejor, depende de la información disponible en las encuestas. Cada método y cada encuesta cuestionan condiciones implícitas o explícitas sobre diferentes factores, ya sean estándares de contratación y/o los requisitos de desempeño. 
1.Autoevaluación de los trabajadores. Se pregunta directamente a los trabajadores sobre los requisitos de escolaridad para su trabajo y el empleado declara si su trabajo coincide o está relacionado con su nivel de educación. Este método ha sido utilizado por Duncan y Hoffman (1991), Sicherman (1991) y Chevalier y Lindley (2009). La desventaja es que se trata de una medida subjetiva y puede diferir entre las personas que realizan el mismo puesto.

2.Análisis de trabajo. Se basa en la información contenida en las clasificaciones ocupacionales. Este método es un examen realizado por analistas ocupacionales concernientes a las cualificaciones requeridas por cada grupo ocupacional y asigna un nivel de educación a cada grupo de acuerdo con la Clasificación internacional uniforme de educación. El diccionario de títulos ocupacionales contiene un indicador de requisitos educativos en la forma de la escala de desarrollo educativo general (GED). Estas categorías de GED se traducen en equivalentes de año escolar. Los trabajadores de un grupo particular que tienen el nivel de educación asignado se consideran bien emparejados. Aquellos que tienen un nivel más alto (inferior) de educación se consideran sobreeducados (subeducados). Algunos de los autores que han utilizado este enfoque son Freeman (1976), Rumberger (1981) y Reis (2017). Algunas de sus desventajas es que no hay consenso sobre la conversión de la escala de GED a años de escolaridad, las actualizaciones son poco frecuentes y no tiene en cuenta la posible diversidad de puestos de trabajo dentro de las categorías ocupacionales más estrechas.

3.Método estadístico. En este método, la cantidad requerida de escolaridad para un trabajador se obtiene del promedio de años de escolaridad de todos los trabajadores que tienen la misma ocupación. Se define a la gente como sobreeducada o subeducada si su nivel completo de escolaridad se dispersa al menos una desviación estándar de la media en su ocupación.

Otra opción es definir la moda de los niveles de escolaridad completados de las personas que trabajan en esa ocupación como el nivel de escolaridad requerido en dicho trabajo. Las personas que tienen más o menos de esta cantidad tienen exceso de escolaridad (sobreeducadas) o falta de escolaridad (subeducadas). Esta medida no utiliza los dos intervalos de desviación estándar alrededor de la medida de centralidad. 
El método estadístico ha sido empleado en Verdugo y Verdugo (1989), Quinn y Rubb (2006), Castillo (2007). Este método tiene la ventaja de que sus medidas de desajuste se obtienen de los mismos datos. Sin embargo, las desventajas son que las medidas derivadas de este método tienden a ser más sensibles a los efectos de las cohortes y al nivel de agregación de la muestra.

\section{TEORÍAS SOBRE DESAJUSTE EDUCATIVO}

La teoría del capital humano afirma que la educación conduce a mayores ganancias a través de su impacto en la productividad. A su vez, la vinculación entre la educación y la productividad se basa en la noción de habilidades. La educación, así como la capacitación formal y la experiencia laboral informal desarrollan o identifican habilidades que hacen que los individuos sean trabajadores más productivos (Becker, 1993).

Varias hipótesis han desafiado la validez de la teoría del capital humano para explicar la discrepancia entre escolaridad y los requisitos laborales. Las principales teorías asociadas al desajuste laboral son: modelos de asignación (Sattinger, 1993); competencia laboral (Thurow, 1975); movilidad profesional (Sicherman y Galor, 1990) y la teoría del matching (Pisarides, 2000). Sin embargo, no hay un acuerdo entre ellas sobre las causas de esta relación observada.

La primera explicación, según Sicherman (1991), se centra en la posible compensación entre escolaridad y otros componentes de capital humano (por ejemplo, los sobreeducados pueden tener menor experiencia en el trabajo o educación de menor calidad o niveles más bajos de inteligencia). Es decir, los trabajadores pueden calificar a trabajos similares al tener diferentes niveles de escolaridad pero niveles similares totales de capital humano total.

La teoría de movilidad laboral de Sicherman y Galor (1990) señala que la sobreeducación no es necesariamente un estado permanente, sostiene que los trabajadores pueden laborar temporalmente en puestos de trabajo que les proporcionen habilidades que luego se utilizarán en una ocupación diferente de mayor nivel. Los individuos adquieren habilidades 
y experiencia en una ocupación para poder moverse a otra ocupación y abandonan la sobreeducación.

La movilidad laboral es una característica sobresaliente del mercado laboral; muy pocos trabajadores realizan la misma tarea a lo largo de sus vidas laborales. Las trayectorias profesionales óptimas de los individuos pueden implicar movilidad intrafirma y movilidad entre empresas.

La movilidad intrafirma (promoción) está sujeta a la decisión del empleador, mientras que la movilidad entre empresas y su momento óptimo están determinadas por las personas que eligen el tiempo de salida óptimo para maximizar sus ganancias esperadas de por vida (Sicherman y Galor, 1990).

Otra explicación se encuentra en el modelo de competencia laboral de Thurow (1975). Esta teoría argumenta que los empleadores usan características personales, como la educación, como criterios en sus decisiones de contratación. La educación es, por lo tanto, un indicador de la cantidad de capacitación que un empleador podría tener para invertir en un empleado. Hay dos colas en el modelo de Thurow (1975). En primer lugar, los trabajadores forman una cola para los trabajos en los que la posición relativa de un trabajador en la cola depende de su nivel de logro educativo. La segunda cola está formada por trabajos clasificados desde los menos exigentes (en términos de capacitación) hasta los que requieren la calificación más alta. Según este modelo, los trabajadores siempre tienen un incentivo para invertir en más educación, ya que los desplaza hacia adelante en la cola de los mejores trabajos. Los trabajos se ordenan con respecto a la capacitación, por lo que el salario ofrecido refleja no solo la productividad, sino también los costos de capacitación de un trabajador en el trabajo. Como los mejores trabajos son escasos, se les asignarán pocos trabajadores y todos los demás con altos niveles de educación serán asignados a empleos de menor calidad que requieren, relativamente, menos educación. Por lo tanto, los empleadores a veces contratan al solicitante con más escolaridad para potencialmente ahorrar en costos de capacitación, independientemente de los requisitos del trabajo.

Un enfoque similar es el de modelos de asignación propuesto por Sattinger (1993), donde los trabajadores con diferentes capacidades se 
asignan a trabajos con diferentes niveles de dificultad o complejidad. La existencia de un problema de asignación implica que los trabajadores enfrentan una opción en su trabajo o sector. La idea básica es que, aunque la educación eleva la productividad en general, el nivel real de productividad realizado también está determinado por la correspondencia entre nivel educativo y nivel de trabajo. La elección del empleo o del sector crea un paso intermedio entre las características de los individuos y sus ingresos.

Los trabajadores que se encuentran en un sector o trabajo en particular no se distribuyen al azar, las ubicaciones de los trabajadores en sectores o empleos se basan en el criterio de que sus opciones maximizan sus ingresos o utilidad. La relación observada se construye a partir de las opciones de los trabajadores.

Por último, la teoría de matching (búsqueda y coincidencia) describe el funcionamiento del mercado de trabajo cuando existe heterogeneidad en los trabajadores y en las empresas y ambos manejan información imperfecta en la búsqueda de empleo (Pissarides, 2000). Los trabajadores buscan ofertas de trabajo en el mercado laboral y las empresas seleccionan el mercado laboral para los trabajadores más productivos. Para ambos lados, la búsqueda es costosa. Por lo tanto, pueden producirse desajustes temporales causados por la insuficiencia (o exceso) de la educación de un trabajador con respecto al trabajo desempeñado o por el nivel de capital humano requerido para el trabajo en cuestión. Ambos tipos de desajuste son eventualmente corregidos, de acuerdo con la teoría de matching, ya que los trabajadores no coincidentes cambian de trabajo para mejorar su coincidencia y obtener un salario más alto.

El presente trabajo plantea la hipótesis que si existe desajuste educativo en el mercado laboral de México y parte del desajuste se encuentra en la heterogeneidad intrínseca en el capital humano de los individuos. Las empresas no solamente valoran el nivel escolar del individuo sino otros aspectos de capital humano como la experiencia laboral y las habilidades de las personas. Sin embargo, la escolaridad excedente no es improductiva si tiene un rendimiento salarial positivo. 


\section{ESTUDIOS PREVIOS DE DESAJUSTE EDUCATIVO}

En los países desarrollados la incidencia y los efectos salariales de los desajustes entre educación y ocupación, específicamente la sobreeducación, están bien documentados en la literatura. El primero en estudiar el tema fue Freeman (1976), cuya motivación fue el elevado aumento en el número de graduados universitarios en la década de 1970 en Estados Unidos, que condujo a una disminución en los rendimientos de la educación universitaria. Freeman (1976) muestra que en un período de tan solo seis años la prima de ingresos de los recién egresados universitarios disminuyó de 40 a 16\%. Este descenso causó preocupaciones sobre la sobreinversión en la educación universitaria en Estados Unidos y surgió la pregunta si esta educación era rentable.

El análisis de Freeman se ajusta al marco neoclásico. El salario universitario disminuye en respuesta a un aumento en la oferta de trabajadores altamente educados. Esto puede suceder porque las empresas ajustan su tecnología de producción para aprovechar el factor de entrada, ahora relativamente más barato y abundante, de los trabajadores altamente calificados. También puede suceder a través de un proceso en el cual los trabajadores altamente educados compiten por un número limitado de empleos calificados al no ofrecer los salarios que demandan. (Leuven y Oosterbeek, 2011).

La literatura de sobreeducación se revitalizó con la publicación del artículo de Duncan y Hoffman (1981). Este estudio trata el tema de sobreeducación a nivel individual al comparar a los trabajadores que terminan en una ocupación que coincide con su nivel de educación adquirida con los trabajadores que terminan en una ocupación que requiere más escolaridad o menos escolaridad de la que adquirieron. Los autores introdujeron una especificación de la ecuación salarial que permite la estimación por separado de los rendimientos de los años de educación requeridos para el trabajo, de los años de sobreeducación y de los años de subeducación.

Ellos encuentran que casi $40 \%$ de la fuerza de trabajo de Estados Unidos (y alrededor de $50 \%$ de los hombres negros) tienen más educación 
de la que requieren sus trabajos. Pero también encuentran que la educación "excedente" sí tiene valor económico.

Según Kucel (2011) , la sobreeducación no es un problema insignificante que afecta solo una minoría de la fuerza de trabajo, su incidencia ha aumentado considerablemente a lo largo de varios países, afecta un cuarto del mercado laboral en economías avanzadas como el Reino Unido, Estados Unidos y Holanda. Leuven y Oosterbeek (2011) resumen los hallazgos de los estudios que han replicado el modelo de Duncan y Hoffman.

Clasifican los resultados por continente, por década de estudio, por método empleado para medir la educación requerida, por método de estimación y por género. El promedio general informado por los estudios existentes es de $30 \%$ de trabajadores sobreeducados y de $26 \%$ de trabajadores subeducados. A su vez, el análisis reporta, que el retorno promedio de un año de escolaridad requerida es de 0.089 , el rendimiento de un año adicional de escolaridad del 0.043 y cada año faltante de educación requerida resulta en una penalización salarial de 0.036 . Es decir, tal como afirman Duncan y Hoffman (1981) la educación excedente no es completamente improductiva, tiene un efecto positivo y significativo en las tasas salariales. Sin embargo, el coeficiente estimado en los años de educación excedente es aproximadamente la mitad del coeficiente en los años requeridos de educación y esto sugiere una posible mala distribución de los recursos educativos.

Del mismo modo, han surgido estudios empíricos para estudiar las causas y efectos del desajuste educativo en el mercado laboral de un país en desarrollo, en Colombia el nivel de desajuste varía entre ocupaciones y grupos de edad (Castillo, 2007); Ecuador presenta 32.52\% de desajuste (Botello, 2016); en Brasil $50 \%$ de la población ocupada presenta desajuste educativo (Reis, 2017); Filipinas, India y Tailandia también presentan niveles de desajuste (Mehta et al. (2011).

Quinn y Rubb (2006) examinan el impacto de los desajustes entre educación y ocupación sobre los salarios en los países en desarrollo, entre ellos México. Sostienen que la educación requerida puede ser dinámica debido a los cambios en la tecnología y la calidad educativa. Por lo tanto, permiten que la educación requerida varíe según el año de nacimiento y el 
año de la encuesta y aproximan el nivel de escolaridad requerido usando el nivel medio de logros para cada clasificación ocupacional. Los autores sugieren, mediante sus resultados empíricos, que un país en desarrollo necesita asegurar que también se produzcan aumentos en los niveles ocupacionales correspondientes para obtener los máximos beneficios económicos de los aumentos en los niveles de educación. De la Garza y Villezca (2006) encuentran que $26 \%$ de las personas con educación superior en México está sobreeducada y obtiene un ingreso $19 \%$ menor al que obtendría si estuviera en una ocupación correcta. Por su parte, Burgos y López (2011) muestran que el nivel de sobreeducación de los egresados de universidades públicas en México es de 22.6 por ciento.

Existen otros estudios que asocian el desajuste educativo con la falta de habilidades. Para Flisi et al (2017) y Chevalier (2003), más educación no se traduce automáticamente en mejores habilidades. Las habilidades individuales son una característica más amplia y más dinámica, ya que se supone que aumentan con la experiencia de trabajo y la capacitación laboral. Además, el nivel real de destrezas que poseen los individuos del mismo nivel educativo puede variar según las diferentes cohortes de edad, debido a los cambios en el sistema educativo y al deterioro del proceso de envejecimiento. Por lo tanto, la medición de las habilidades de los adultos en lugar del logro educativo se considera un enfoque superior y más confiable para cuantificar las competencias reales de un individuo en un momento específico. Flisi et al (2017) utilizan la encuesta PIAAC (por sus siglas en inglés) para medir el desajuste profesional en 17 países europeos a nivel individual, basado en las variables educativas (sobreeducación) y basadas en habilidades (exceso de habilidades) usando medidas objetivas y subjetivas. Encuentran que $10 \%$ de los trabajadores sobreeducados no cuentan con las habilidades requeridas en su trabajo, mientras que $13 \%$ de trabajadores exhiben solamente desigualdad educativa y $20 \%$ únicamente desajuste de habilidades.

Chevalier y Lindley (2009), por su parte, estudian el tema de la sobreeducación en Reino Unido antes y después de la expansión de graduados en la década de 1990. Sus resultados muestran que la sobreeducación surge de la heterogeneidad en las habilidades de los egresados. En general, 
no está asociada con una escasez de habilidades académicas, pero se correlaciona con la reducción de las habilidades del mercado de trabajo y las características no observadas desfavorables.

Aunque los estudios empíricos iniciales de sobreeducación abordaron el fenómeno desde una perspectiva económica, investigaciones más recientes han analizado el tema desde una óptica sociológica (Kucel (2011) y Capsada Munsech (2017). Su preocupación principal es colocar la sobreeducación en el centro y enfocarse en sus consecuencias en términos de estratificación social. Buscan explicar si los trabajadores sobreeducados lo ven como un trampolín o una trampa, y hasta qué punto esto varía entre individuos con diferentes características.

Una diferencia importante entre los artículos que miden el desajuste educativo es la muestra utilizada de trabajadores, hay quienes utilizan empleados en general con independencia de su nivel escolar y otros emplean solo muestras de graduados de nivel superior. La división entre las encuestas de graduados y de la población general demuestra ser la dimensión clave para la medición de la magnitud de la incidencia de la sobreeducación (los estudios basados en encuestas de postgrado no son representativos a nivel nacional; están restringidos a sus grupos objetivo específicos).

Esta diferencia podría atribuirse directamente a las distintas formas de medición. La sobreeducación entre la población trabajadora total se mide, generalmente, con métodos estadísticos para cada ocupación, mientras que para los graduados de posgrado se detecta a través de la autoevaluación de los trabajadores (Kucel, 2011).

En general, los resultados de todos los estudios existentes de desajuste educativo muestran que la incidencia de este fenómeno difiere mucho con base en la conceptualización, medición de educación requerida y el modelo estimado.

\section{DATOS Y METODOLOGÍA}

Se emplea la base de datos de la encuesta nacional sobre niveles de vida de los hogares (ENNViH), una encuesta de carácter longitudinal, multitemática, representativa de la población mexicana a nivel nacional, urbano, rural 
y regional. Se emplea información de un periodo de diez años, recopilada a través de dos levantamientos instrumentados en 2002 y 2009-2012. Las variables a utilizar son: años de escolaridad, nivel de escolaridad, experiencia real, experiencia al cuadrado, ocupación, salario, resultado del test de habilidad, ingreso no laboral, dummies de género, estado civil y jefe de hogar.

En primer lugar, se utilizan como muestra solamente a las personas entre 15 y 65 años de edad del último levantamiento (2009-2012), que declararon estar trabajando y que reciben un salario. Es decir, no se contemplan los trabajadores por cuenta propia, trabajadores sin remuneración ni los patrones. Se obtiene el porcentaje de desajuste educativo de estos trabajadores y su efecto en los salarios. Posteriormente, se utilizan la muestra de personas que se entrevistaron tanto en el año 2002 como en el 2012 para verificar si su estado de desajuste cambió o permaneció igual en el transcurso de ese periodo.

En el cuadro 1 se presenta la información referente a la educación requerida por nivel ocupacional utilizando dos enfoques:

-Una medida estadística con medidas de tendencia central: la media y la moda.

-Una medida normativa basada en la clasificación internacional uniforme de ocupaciones (CIUO-88)

Se puede observar que el nivel de escolaridad requerida varía según el enfoque utilizado. Sin embargo, para este trabajo se emplea el método estadístico basado en la escolaridad promedio. 


\begin{tabular}{|c|c|c|c|c|}
\hline \multicolumn{5}{|c|}{$\begin{array}{l}\text { Cuadro } \mathbf{1} \\
\text { Escolaridad requerida por ocupación }\end{array}$} \\
\hline \multirow[b]{2}{*}{ Ocupación } & \multicolumn{3}{|c|}{ Método estadístico } & \multirow{2}{*}{$\begin{array}{c}\text { Método normativo } \\
\text { Diccionario de títulos } \\
\text { ocupacionales }\end{array}$} \\
\hline & Media & desv. est. & Moda & \\
\hline Agricultura, ganadería, caza y pesca & 5.5 & 3.5 & 6 & Secundaria $(9)$ \\
\hline Apoyo administrativo & 11.5 & 2.9 & 12 & Preparatoria (12) \\
\hline Arte, espectáculos y deporte & 11.3 & 3.6 & 9 & Lic. corta o TSU (14) \\
\hline Artesanos & 7.4 & 2.7 & 9 & Secundaria (9) \\
\hline Ayudantes y peones & 9.1 & 3.6 & 9 & Primaria (6) \\
\hline Comerciantes, empleados de comercio & 9.6 & 3.2 & 9 & Preparatoria (12) \\
\hline Conductores maquinaria y transporte & 7.8 & 3.1 & 9 & Secundaria (9) \\
\hline Funcionarios y directivos & 9.0 & 3.8 & 9 & Licenciatura (16) \\
\hline $\begin{array}{l}\text { Jefes de departamento, coordinadores, } \\
\text { área admin. }\end{array}$ & 12.2 & 3.3 & 16 & Lic. corta o TSU (14) \\
\hline Operadores de maquinaria fija & 8.3 & 3.0 & 9 & Secundaria (9) \\
\hline Profesionistas & 12.9 & 3.4 & 16 & Lic. o posgrado $(>=16)$ \\
\hline Protección, monitoreo y fuerzas armadas & 8.7 & 3.7 & 9 & Secundaria (9) \\
\hline Servicios domésticos & 6.5 & 3.5 & 9 & Primaria (6) \\
\hline Servicios profesionales & 8.1 & 3.5 & 9 & Preparatoria (12) \\
\hline Técnicos & 7.7 & 3.6 & 9 & Lic. corta o TSU (14) \\
\hline Trabajadores de la educación & 15.0 & 2.8 & 16 & Lic. o posgrado $(>=16)$ \\
\hline Vendedores ambulantes & 8.3 & 3.1 & 9 & Primaria (6) \\
\hline
\end{tabular}

El cuadro 2 emplea el método estadístico (se considera como medida central la media) para obtener el porcentaje de desajuste educativo en el mercado laboral de México, la proporción de trabajadores sobreeducados y subeducados. Si el individuo tiene un nivel educativo superior a la media más o menos una desviación estándar, se considera que es sobreeducado o subeducado, respectivamente. Se encontró que en general existe $26.3 \%$ de desajuste educativo en los trabajadores mexicanos, solo $10.5 \%$ de los trabajadores se encuentran sobreeducados y $16.3 \%$ subeducado. No obstante, dicho porcentaje varía si analizamos por separado cada ocupación. Por ejemplo, $34.12 \%$ de las personas que trabajan como jefes de departamento 


\begin{tabular}{|l|c|c|c|c|}
\hline \multicolumn{5}{|c|}{$\begin{array}{c}\text { Cuadro 2 } \\
\text { Desajuste educativo por el método estadístico (Media) }\end{array}$} \\
\hline \multicolumn{1}{|c|}{ Ocupación } & Bien asignado & Sobreeducado & Subeducado & $\begin{array}{c}\% \text { del total } \\
\text { de obs. }\end{array}$ \\
\hline Agricultura, ganaderí, caza y pesca & 70.70 & 9.73 & 19.57 & 10.88 \\
\hline Apoyo administrativo & 67.37 & 20.34 & 12.29 & 3.73 \\
\hline Arte, espectáculos y deporte & 71.06 & 22.05 & 6.89 & 0.16 \\
\hline Artesanos & 73.90 & 9.78 & 16.32 & 0.46 \\
\hline Ayudantes y peones & 82.07 & 10.07 & 7.86 & 4.12 \\
\hline Comerciantes, empleados de comercio & 64.72 & 15.82 & 19.46 & 8.59 \\
\hline Conductores maquinaria móvil y & 78.04 & 12.23 & 9.73 & 4.4 \\
\hline transporte & & & & \\
\hline Funcionarios y directivos & 81.0 & 13.84 & 5.16 & 0.62 \\
\hline Jefes de departamento y coordinadores & 60.79 & 34.12 & 5.09 & 1.58 \\
\hline Operadores de maquinaria fija & 72.99 & 13.99 & 13.02 & 9.96 \\
\hline Profesionistas & 72.63 & 5.49 & 21.88 & 17.56 \\
\hline Protección, monitoreo y fuerzas armadas & 79.55 & 7.33 & 13.12 & 3.76 \\
\hline Servicios domésticos & 63.49 & 8.05 & 28.46 & 5.67 \\
\hline Servicios profesionales & 80.12 & 7.36 & 12.53 & 7.62 \\
\hline Técnicos & 76.83 & 6.12 & 17.05 & 13.33 \\
\hline Trabajadores de la educación & 83.76 & 0.0 & 16.24 & 6.67 \\
\hline Vendedores ambulantes & 64.98 & 25.52 & 9.50 & 0.89 \\
\hline Total de desajuste & 73.2 & 10.5 & 16.3 & 100 \\
\hline Observaciones expandidas & $16,603,266$ & $2,382,063$ & $3,430,493$ & $22,415,822$ \\
\hline Fuente: elaboración propia con datos de ENNViH & & & & \\
\hline
\end{tabular}

y coordinadores tienen más años de escolaridad del que requiere su trabajo y los artesanos solo $9.78 \%$ se encuentra en esta situación.

Por género, afecta más a las mujeres que a los hombres, pero hay un porcentaje mayor de hombre sobreeducados con respecto a las mujeres (cuadro 3). Por grupos de edad, los más jóvenes presentan mayores niveles de sobreeducación $(14.6 \%)$ y $46.5 \%$ de los que tienen edad entre 56-65 años se consideran subeducados. Asimismo, los trabajadores con posgrado y con universidad incompleta suelen padecer mayor sobreeducación. 6 . 


\begin{tabular}{|c|c|c|c|c|c|}
\hline \multicolumn{6}{|c|}{$\begin{array}{c}\text { Cuadro } 3 \\
\text { Desajuste educativo según características personales }\end{array}$} \\
\hline \multicolumn{2}{|r|}{ Variable } & Desajuste & Sobreeducado & Subeducado & $\%$ del total \\
\hline \multirow[t]{2}{*}{ Género } & Hombre & 27.7 & 13.0 & 14.8 & $63.9 \%$ \\
\hline & Mujer & 30.1 & 11.0 & 19.2 & $36.1 \%$ \\
\hline \multirow{5}{*}{$\begin{array}{l}\text { Grupos de } \\
\text { edad (años) }\end{array}$} & $15-25$ & 22.5 & 14.6 & 7.9 & $27.4 \%$ \\
\hline & $26-35$ & 25.5 & 14.0 & 11.5 & $28.1 \%$ \\
\hline & $36-45$ & 26.7 & 10.5 & 16.2 & $23.6 \%$ \\
\hline & $46-55$ & 38.6 & 10.2 & 28.4 & $14.4 \%$ \\
\hline & $56-65$ & 52.4 & 5.9 & 46.5 & $6.5 \%$ \\
\hline \multirow{7}{*}{ Escolaridad } & Sin instrucción & 75.1 & 0.0 & 75.1 & $13.2 \%$ \\
\hline & Primaria & 11.8 & 0.0 & 11.8 & $18.3 \%$ \\
\hline & Secundaria & 11.1 & 0.0 & 11.1 & $33.9 \%$ \\
\hline & Preparatoria & 20.6 & 15.7 & 5.0 & $14.2 \%$ \\
\hline & Universidad incompleta & 28.9 & 28.9 & 0.0 & $4.4 \%$ \\
\hline & Universidad & 27.6 & 27.6 & 0.0 & $14.3 \%$ \\
\hline & Posgrado & 61.1 & 61.1 & 0.0 & $1.7 \%$ \\
\hline \multirow[t]{2}{*}{ Area } & Urbana & 26.4 & 10.8 & 15.6 & $59.1 \%$ \\
\hline & Rural & 29.3 & 9.9 & 19.4 & $40.9 \%$ \\
\hline
\end{tabular}

\section{MODELOS ECONOMÉTRICOS}

\subsection{Modelos de determinantes del desajuste educativo}

Diversos estudios estiman modelos probit de los determinantes de la sobreeducación y subeducación. La especificación de estos modelos varía entre autores, pero coinciden en que los jóvenes, las mujeres, los inmigrantes y las personas que no están casadas tienen más probabilidades de estar sobreeducados (Leuven y Oosterbeek, 2011; Castillo, 2007; Quinn y Rubb, 2006):

a)Género. Cuando los hombres son los principales proveedores de ingresos en el hogar, las mujeres están, necesariamente, más restringidas, y esto puede traducirse en una mayor probabilidad de estar sobreeducada.

b)Edad. Los trabajadores mayores tienen menos probabilidades de ser sobreeducados que sus colegas más jóvenes (promoción laboral, experiencia laboral). 
c)Etnicidad. Cuando las minorías no han sido educadas en otros países, pueden estar restringidos geográficamente o tener niveles bajos de capital humano (o discriminación).

d)Habilidad. Otro determinante de estar sobreeducado puede ser un nivel más bajo de habilidad. Los estudios que tienen acceso a medidas de capacidad (Chevalier y Lindley, 2009; Allen y van der Velden (2001) encuentran que la capacidad y la sobreeducación están negativamente correlacionadas.

\subsection{Modelos de incidencia en los salarios}

Las investigaciones acerca de desajuste laboral han empleado dos tipos de modelos para estimar los efectos salariales de la sobreeducación y subeducación: el modelo propuesto por Duncan y Hoffman (1981) que permite estimar el retorno de un año de educación o subeducación adicional y los modelos basados en variables dummy que solo permiten la estimación de la pérdida de oportunidad relativa asociada al desajuste (Kucel, 2011). Ambos enfoques son de uso común de acuerdo con la pregunta de investigación.

$$
\ln \left(W_{i}\right)=X_{i} \beta+\propto S_{i}^{r}+\delta S_{i}^{0}+\tau S_{i}^{u}+\varepsilon_{i}
$$

Si seguimos el modelo propuesto por Duncan y Hoffman (1981), la primera especificación utilizada para estimar los efectos salariales del desajuste educativo puede escribirse de la siguiente manera:

Donde $\mathrm{W}_{\mathrm{i}}$ corresponde al salario individual del trabajador, $\mathrm{X}_{\mathrm{i}}$ es un vector de características individuales que incluyen experiencia y experiencia al cuadrado. La aportación de Duncan y Hoffman es que fraccionan el total de años de escolaridad de un individuo(S) en tres componentes: escolaridad requerida por el trabajo $\left(\mathrm{S}^{\mathrm{r}}\right)$, escolaridad del trabajador excedente a la requerida $\left(\mathrm{S}^{0}\right)$ y escolaridad faltante a la requerida $\left(\mathrm{S}^{\mathrm{u}}\right)$.

$\mathrm{S} \equiv \mathrm{S}^{\mathrm{r}}+\mathrm{S}^{\mathrm{o}}-\mathrm{S}^{\mathrm{u}}$ 
La interpretación de los coeficientes son los siguientes:

$\propto$ :EL retorno a la escolaridad requerida en el puesto de trabajo.

$\delta$ : El rendimiento de un año adicional de escolaridad que excede el requisito de trabajo, en relación con los compañeros de trabajo (los trabajadores con la misma escolaridad requerida que tiene el nivel requerido de enseñanza).

$\delta$ - $\propto$ : El rendimiento de un año adicional de escolaridad que excede el requisito de trabajo, en relación con los trabajadores con el mismo nivel de escolaridad que tienen la escolaridad requerida en el trabajo.

$\tau$ : La pérdida de salario debido a un año de educación faltante, en relación con los compañeros de trabajo (con la misma escolaridad requerida) y $\propto+\tau$ : Las diferencias salariales entre los trabajadores que trabajan en puestos que requieren un año adicional de escolaridad (un año más de lo que tienen) y los trabajadores que tienen el mismo nivel de escolaridad pero trabaja en puestos que requieren ese nivel de escolaridad.

$$
\ln \left(W_{i}\right)=X_{i} \beta+\delta(O V E R)+\tau(U N D E R)+\varepsilon_{i}(2)
$$

Por otra parte, para comparar los trabajadores de una misma ocupación con iguales características individuales, pero que solo difieren en su nivel de escolaridad, la siguiente ecuación proporciona una comparación apropiada y directa:

Donde:

$$
\begin{aligned}
& O V E R=\left\{\begin{array}{cc}
1 & \text { si el trabajador es sobreeducado } \\
0 & \text { si tiene la escolaridad requerida o es subeducado }
\end{array}\right\} \\
& U N D E R=\left\{\begin{array}{lc}
1 & \text { si el trabajador es subeducado } \\
0 & \text { si tiene la escolaridad requerida o es sobreducado }
\end{array}\right\}
\end{aligned}
$$

El presente trabajo presenta estimación y resultados de ambos modelos. 


\section{ESTIMACIÓN Y RESULTADOS}

El análisis en este documento se realiza en dos partes. Primero se estima un modelo logit multinomial para encontrar los posibles determinantes del desajuste educativo independientes a la escolaridad.

$$
\operatorname{Pr}\left(Y_{i}=j\right)=\beta_{1} \text { Raven }+\beta_{2} \text { experiencia }_{i}+\beta_{3} \text { experiencia }_{i}{ }^{2}+\beta_{4} X_{i}+\varepsilon_{i} \text { (3) } \mathrm{j}=
$$

Donce:

$$
3
$$

$\mathrm{Y}=$ tipo de desajuste educativo, $\mathrm{Y}=1$ adecuadamente educado para su ocupación, $Y=2$ sobreeducado, $Y=3$ subeducado, $X$ es un vector de variables de control que incluye género, ingreso no laboral del hogar, estado civil y zona de residencia.

El cuadro 4 muestra los resultados del modelo que corresponde a la probabilidad de un individuo de ser sobreeducado o subeducado (en comparación con estar adecuadamente educado).

$$
\begin{aligned}
& \log \left[\frac{\operatorname{Pr}(\text { sobreeducado })}{\operatorname{Pr}(\text { Adecuado })}\right]=\beta_{1} \text { Raven }+\beta_{2} \text { experiencia }+\beta_{3} \text { experiencia }{ }^{2}+\beta_{4} X+\varepsilon(3.1) \\
& \log \left[\frac{\operatorname{Pr}(\text { subeducado })}{\operatorname{Pr}(\text { Adecuado })}\right]=\beta_{1} \text { Raven }+\beta_{2} \text { experiencia }+\beta_{3} \text { experiencia }^{2}+\beta_{4} X+\varepsilon(3.2)
\end{aligned}
$$

Ambas regresiones incluyen experiencia laboral, experiencia al cuadrado, $\mathrm{X}$ es un vector de variables de control que incluye género, ingreso no laboral del hogar, los resultados de la prueba de habilidades Raven, estado civil y zona de residencia. También se controla por años de escolaridad.

Los resultados del cuadro 4 indican que la experiencia disminuye la probabilidad de que un individuo esté sobreeducado (relativo a ser adecuadamente educado), ceteris paribus, y a su vez, la experiencia aumenta la probabilidad de que un individuo esté subeducado (en relación con ser educado adecuadamente). Por ejemplo, los datos de la columna (a) señalan que un año más de experiencia laboral reduce la probabilidad de que la persona esté sobreeducada en lugar de estar bien asignada en $3.15 \%$; por lo tanto, la experiencia laboral sustituye, en algunos casos, a los requisitos 


\begin{tabular}{|c|c|c|c|c|c|c|}
\hline \multicolumn{7}{|c|}{$\begin{array}{c}\text { Cuadro } 4 \\
\text { Resultados modelo logit }\end{array}$} \\
\hline & \multicolumn{3}{|c|}{ (a) } & \multicolumn{3}{|c|}{ (b) } \\
\hline & \multirow{2}{*}{\multicolumn{3}{|c|}{$\log \left[\frac{\operatorname{Pr}(\text { sobreeducado })}{\operatorname{Pr}(\text { Adecuado })}\right]$}} & \multicolumn{3}{|c|}{$[\operatorname{Pr}($ subeducado $)]$} \\
\hline & & & & \multicolumn{3}{|c|}{$[\overline{\operatorname{Pr}(\text { Adecuado })}]$} \\
\hline Variables & \multicolumn{2}{|c|}{ Coeficiente } & Z-estadist & \multicolumn{2}{|c|}{ Coeficiente } & \multirow{2}{*}{$\begin{array}{r}\text { Z-estadist } \\
2.16\end{array}$} \\
\hline Experiencia & -0.0315 & $* * *$ & -2.79 & 0.0219 & $* *$ & \\
\hline Experiencia2 & 0.0004 & & 1.28 & -0.0003 & * & -1.7 \\
\hline $\begin{array}{l}\text { Ingreso no laboral del } \\
\text { hogar (miles/mes) }\end{array}$ & -0.0040 & $* *$ & -2.28 & 0.0029 & $* * *$ & 2.49 \\
\hline Género (1=mujer $)$ & -0.4737 & $* * *$ & -5.82 & 0.3242 & $*$ & 3.51 \\
\hline Zona (1=Rural) & 0.0941 & & 1.46 & -0.1360 & $* *$ & -2.16 \\
\hline Raven & -0.0686 & $*$ & -1.8 & 0.0590 & $*$ & 1.78 \\
\hline Casado & 0.0252 & & 0.34 & 0.0610 & & 0.69 \\
\hline Jefe_hogar & -0.0221 & & -0.26 & -0.0204 & & -0.25 \\
\hline Constante & -2.3009 & & -18.91 & 0.4181 & & 2.34 \\
\hline
\end{tabular}

educativos al momento de buscar un trabajo. Si los trabajadores cuentan con mil pesos más de ingreso no laboral en el hogar se reduce su probabilidad de ser sobreeducado en comparación de bien asignado en 0.4 por ciento. Es decir, entre mayor sea el ingreso no laboral de las personas, éstas podrían estar dispuestas a esperar más hasta encontrar un trabajo que si le corresponda a su escolaridad.

El estado civil y la zona de residencia no tienen un impacto estadísticamente significativo sobre la probabilidad de sobreeducación y subeducación de los trabajadores. La prueba de habilidades muestra una relación negativa con la probabilidad de ser sobreeducado (en relación con ser adecuadamente educado para su ocupación), es decir, que las habilidades también juegan un papel importante al momento de conseguir un puesto de trabajo, entre mayor sea tu nivel de habilidad menor será la probabilidad de ser sobreeducado (6.8\% menos). De igual manera, entre mayor sea el nivel de habilidad de las personas, mayor es su probabilidad de ser subeducado, esto es, conseguir un empleo para el cual no cuente con educación requerida.

En general, los resultados indican que los trabajadores sobreeducados es más probable que sean hombres, tengan menos experiencia, posean mayor escolaridad pero un nivel menor de habilidades. Por otro lado, los 
subeducados es más probable que sean mujeres, tengan menos escolaridad y mayor experiencia y nivel de habilidades, vivan en una zona urbana.

Aunque datos anteriores muestran que existe $10.5 \%$ de sobreeducación, no significa, necesariamente, que estos años de exceso de educación no tengan ningún valor económico para las personas. El presente artículo utiliza dos enfoques para modelar el efecto salarial del desajuste educativo. El primer enfoque sigue la especificación de Mincer (1974) y se incluyen variables dummies por desajuste educativo. El segundo enfoque sigue el modelo de Duncan y Hoffman (1981) explicado en el apartado 6.2. La variable dependiente en ambos modelos es el logaritmo natural del salario por hora y las variables independientes incluyen años de escolaridad, experiencia laboral real y experiencia al cuadrado, género, resultados de la prueba de habilidad y una variable de zona de residencia.

El principal interés se encuentra en las diferencias entre los coeficientes estimados en la educación requerida y los de años de ecuación con superávit o déficit. De acuerdo con Duncan y Hoffman (1981), si los niveles de productividad y los salarios en el empleo son inflexibles, entonces los coeficientes estimados para la sobreeducación y subeducación deberían ser cero. Sin embargo, si los niveles de productividad en el trabajo son más variables y si la productividad del trabajador está relacionada positivamente con el nivel de educación sin tener en cuenta los requisitos, entonces se espera un coeficiente positivo en años de escolaridad excedentes y uno negativo en años de educación deficitaria.

El cuadro 5 muestra los resultados de estas regresiones. La columna (a) presenta los resultados de la ecuación salarial minceriana estándar. Los resultados son los esperados, para cada año adicional de escolaridad se incrementa el salario por hora esperado en $10.04 \%$, se incluye una variable proxy de habilidades para corregir el sesgo por habilidades innatas. La variable de habilidades también resulta positiva estadísticamente significativa con un coeficiente de 7.15 por ciento.

La columna (b) muestra los resultados para los retornos asociados a la ecuación de Duncan y Hoffman. Cada año de escolaridad requerida incrementa el salario 15.48 por ciento. Cabe señalar que la escolaridad más 
allá de la requerida no es improductiva, para los años de sobreeducación, el retorno de los salarios es solamente un poco menor que el retorno de la escolaridad requerida $14.66 \%$, es decir, los años excedentes al estar sobreeducados tienen un rendimiento casi equivalente al que obtendría estando

\begin{tabular}{|c|c|c|c|}
\hline \multicolumn{4}{|c|}{ Cuadro 5} \\
\hline \multicolumn{4}{|c|}{ Estimaciones de salarios } \\
\hline & (a) & (b) & (c) \\
\hline & Mincer & $\begin{array}{l}\text { Duncan y } \\
\text { Hoffman }\end{array}$ & $\begin{array}{c}\text { Dummies de } \\
\text { desajuste }\end{array}$ \\
\hline \multirow[t]{2}{*}{ Años de escolaridad } & $0.1004 * * *$ & & $0.1215^{* * *}$ \\
\hline & $(23.29)$ & & $(25.42)$ \\
\hline \multirow[t]{2}{*}{ Años de escolaridad requerida } & & $0.1548 * * *$ & \\
\hline & & $(25.37)$ & \\
\hline \multirow[t]{2}{*}{ Años de "sobreeducación" } & & $0.1466 * * *$ & \\
\hline & & $(7.89)$ & \\
\hline \multirow[t]{2}{*}{ Años de "subeducación" } & & $-0.0625 * * *$ & \\
\hline & & $(-4.13)$ & \\
\hline \multirow[t]{2}{*}{ "Sobreeducado"(1=si) } & & & $-0.2518 * * *$ \\
\hline & & & $(-5.91)$ \\
\hline \multirow[t]{2}{*}{ "Subeeducado" (1=si) } & & & $0.3673 * * *$ \\
\hline & & & $(8.44)$ \\
\hline \multirow[t]{2}{*}{ Raven } & $0.0715^{* * *}$ & $0.0794 * * *$ & $0.0643 * * *$ \\
\hline & $(4.91)$ & $(5.76)$ & $(4.55)$ \\
\hline \multirow[t]{2}{*}{ Experiencia } & $0.0292 * * *$ & $0.0249 * * *$ & $0.0276 * * *$ \\
\hline & $(9.46)$ & $(7.91)$ & $(9.07)$ \\
\hline \multirow[t]{2}{*}{ Experiencia $^{2}$} & $-0.0003 * * *$ & $-0.0004 * * *$ & $-0.0003 * * *$ \\
\hline & $(-4.92)$ & $(-5.83)$ & $(-5.08)$ \\
\hline $\mathrm{R}^{2} \_$adj & 0.287 & 0.322 & 0.313 \\
\hline Núm. de observaciones & 5816 & 5816 & 5816 \\
\hline
\end{tabular}

en un trabajo bien asignado. Las estimaciones de los coeficientes de subeducación es negativa, es decir que, por cada año faltante de escolaridad requerida, los trabajadores obtienen una penalización de su salario de $6.25 \%$ menos de lo que recibirían si estuvieran adecuadamente educados.

Los salarios no parecen estar únicamente sobre la base de los requisitos educativos. La educación excedente y las habilidades tienen un efecto 


\begin{tabular}{|c|c|c|}
\hline \multicolumn{3}{|r|}{ Cuadro 6} \\
\hline \multicolumn{3}{|r|}{$\begin{array}{l}\text { Resumen de coeficientes } \\
\text { (Rendimiento con desajuste educativo) }\end{array}$} \\
\hline Coeficiente & Valor & Interpretación \\
\hline$\alpha$ & 0.1548 & $\begin{array}{l}\text { Por cada año de educación requerida en el trabajo se te otorga un } \\
\text { rendimiento del } 15.48 \% \text { adicional de ingreso. }\end{array}$ \\
\hline$\delta$ & 0.1466 & $\begin{array}{l}\text { Por cada año de escolaridad adicional a la requerida en el puesto de } \\
\text { trabajo obtienes un rendimiento del } 14.66 \% \text { más de ingreso. }\end{array}$ \\
\hline$\tau$ & -0.0625 & $\begin{array}{l}\text { Por cada año de escolaridad faltante a la requerida por tu puesto de } \\
\text { trabajo obtienes una penalización de } 6.25 \% \text { de ingreso. }\end{array}$ \\
\hline$\delta-\alpha$ & 0.0082 & $\begin{array}{l}\text { Muestra la pérdida de } 0.8 \% \text { ingreso de un trabajador por cada año } \\
\text { adicional de sobreeducación comparado con otro que, con el mismo } \\
\text { nivel educativo, ha conseguido un ajuste correcto entre la educación } \\
\text { alcanzada y la requerida en su empleo. }\end{array}$ \\
\hline$\tau+\alpha$ & 0.0923 & $\begin{array}{l}\text { Indica el salario adicional ( } 9 \% \text { ) que recibe un individuo por cada año } \\
\text { de subeducación en comparación con los trabajadores que, con su } \\
\text { mismo nivel formativo, han conseguido un empleo para el que } \\
\text { presentan un ajuste correcto. }\end{array}$ \\
\hline
\end{tabular}

positivo y significativo en las tasas salariales. Además, los trabajadores que tienen empleos para los cuales no están calificados ganan menos que los individuos que si cuentan con la escolaridad requerida. El cuadro 6 muestra la interpretación de los coeficientes obtenidos estimando el modelo Duncan y Hoffman (1981).

Se puede analizar que, en realidad, la pérdida salarial de una persona sobreeducada no es alta con relación a si estuviera en un trabajo que corresponda a sus años de escolaridad, por cada año excedente solamente pierde $.8 \%$ de salario. En cambio, la ganancia de estar subeducado en relación con no estarlo es $9 \%$ de salario adicional por cada año de subeducación.

Por último, en el cuadro 7 se hace una comparación del estatus de una muestra de individuos (2 881) que fueron entrevistados en ambos levantamientos de la encuesta de la ENNViH. El porcentaje de desajuste es, aproximadamente, igual en ambos años. Sin embargo, se observa que del total de personas que se encontraban sobreeducadas en el año 2002, 49 


\begin{tabular}{|c|c|c|c|c|}
\hline \multicolumn{5}{|c|}{$\begin{array}{c}\text { Cuadro } 7 \\
\text { Cambio del estado de desajuste educativo entre la primera y la tercer } \\
\text { entrevista (porcentaje) }\end{array}$} \\
\hline \multirow[t]{2}{*}{ Año } & \multirow[b]{2}{*}{ Desajuste } & \multicolumn{3}{|c|}{2009} \\
\hline & & $\begin{array}{l}\text { Subeducado } \\
(17 \%)\end{array}$ & $\begin{array}{l}\text { Bien } \\
\text { asignado } \\
(69 \%)\end{array}$ & $\begin{array}{c}\text { Sobreeducado } \\
(14 \%)\end{array}$ \\
\hline \multirow[t]{6}{*}{2002} & \multirow{2}{*}{$\begin{array}{l}\text { Subeducado } \\
(15 \%)\end{array}$} & 840,281 & 546,578 & 20,741 \\
\hline & & $8.8 \%$ & $6 \%$ & $0.2 \%$ \\
\hline & \multirow{2}{*}{$\begin{array}{c}\text { Bien asignado } \\
(71 \%)\end{array}$} & 705,022 & $5,407,797$ & 641,459 \\
\hline & & $7.4 \%$ & $57 \%$ & $6.8 \%$ \\
\hline & \multirow{2}{*}{$\begin{array}{c}\text { Sobreeducado } \\
(14 \%)\end{array}$} & 21,839 & 644,365 & 646,903 \\
\hline & & $0.2 \%$ & $6.8 \%$ & $6.8 \%$ \\
\hline \multicolumn{5}{|c|}{ Observaciones expandidas $=9,474,985$} \\
\hline \multicolumn{5}{|c|}{$73 \%$ permanecieron igual y $13 \%$ mejoraron su situación } \\
\hline \multicolumn{5}{|c|}{ Fuente: elaboración propia con datos de la ENNVIH I y ENNVIH III } \\
\hline
\end{tabular}

$\%$ se encuentran bien asignadas para el año 2012. Lo mismo sucede para $39 \%$ de trabajadores que en el año 2002 se encontraban en un estado de subeducados. En general, $73 \%$ de los individuos mantuvieron el mismo estatus de desajuste durante el periodo de análisis y $13 \%$ mejoró su situación. Es decir, un bajo porcentaje de personas dejan de encontrarse con desajuste educativo con el paso del tiempo.

\section{CONCLUSIONES}

Con el presente trabajo se ha tratado de contribuir a la línea de investigación sobre desajuste educativo. En general, se encontró que este fenómeno existe en $26.3 \%$ de los trabajadores de México. Los resultados indican que los trabajadores sobreeducados es más probable que sean hombres, tengan menos experiencia, posean mayor escolaridad pero un nivel menor de habilidades. Por otro lado, los subeducados es más probable que sean mujeres, tengan menos escolaridad y mayor experiencia y nivel de habilidades, vivan en una zona urbana. Los resultados apuntan que una de las posibles causas del desajuste podría ser una compensación entre la escolaridad y 
otras formas de capital humano (experiencia, habilidad) para que los trabajadores puedan calificar a trabajos similares al tener diferentes niveles de escolaridad. Es decir, al momento de contratar una persona en el mercado laboral mexicano no solo se toman en cuenta los requisitos de años de escolaridad sino también otros aspectos de capital humano.

Asimismo, al estudiar el grado de ajuste entre la educación recibida por el trabajador y la requerida en el puesto de trabajo se observa que el rendimiento asociado a la escolaridad difiere entre años de escolaridad requerida, años de escolaridad excedente y años de escolaridad faltante, destacando que es superior el retorno a la escolaridad requerida, pero tan solo son un punto porcentual superior a la escolaridad excedente, lo que sugiere que la educación extra de estos individuos no es improductiva.

Además, los salarios no parecen estar únicamente sobre la base de los requisitos educativos. La experiencia laboral y las habilidades tienen un efecto positivo y significativo en las tasas salariales.

\section{REFERENCIAS}

Allen, J., y van der Velden, R.. (2001). Educational Mismatches versus Skill Mismatches: Effects on Wages, Job Satisfaction, and On-the-Job Search. Oxford Economic Papers, 53(3), 434-452.

Becker, G. 1993. Human Capital: A theoretical and Empirical Analysis, with Special Reference to Education, Chicago: University of Chicago Press, $3^{\mathrm{a}}$. ed.

Botello Peñaloza, H. A. 2016. Determinantes y efectos del desajuste educativo en el mercado laboral ecuatoriano 2007-2012. Investigación y Desarrollo, 24(2), 307-328.

Burgos Flores, B., y López Montes, K. (2011). Efectos de la sobreeducación y el desfase de conocimientos sobre los salarios y la búsqueda de trabajo de profesionistas: Resultados de un estudio basado en opiniones y 
percepciones de egresados universitarios y empleadores. Perfiles educativos, 33(134), 34-51

Capsada Munsech, Q. 2017. Overeducation: Concept, theories, and empirical evidence, Sociology Compass, 11(10).

Castillo Caicedo, M. 2007. Desajuste educativo por regiones en Colombia: ¿competencia por salarios o por puestos de trabajo? Cuadernos de Economía, 26(46): 107-145.

Chevalier, A. 2003. Measuring over education, Economica, 70(279): 509531.

Chevalier, A. y J. Lindley. 2009. Overeducation and the Skills of UK Graduates, Journal of the Royal Statistical Society, Series A, 172(2): 307-337.

De la Garza Garza, O.J. y P.A. Villezca Becerra (2006), Efecto de la sobreeducación en el ingreso de las personas con estudios de nivel superior en México, Ensayos, XXV(2): 21-52.

Duncan, G. J., y Hoffman, S. D. 1981. The incidence and wage effects of overeducation, Economics of Education Review, 1(1), 75-86.

ENNVIH. Encuesta Nacional sobre Niveles de Vida de los Hogares. http:// www.ennvih-mxfls.org/

ENNVIH I. Encuesta Nacional sobre Niveles de Vida de los Hogares (2002). http://www.ennvih-mxfls.org/ennvih-1.html

ENNVIH III. Encuesta Nacional sobre Niveles de Vida de los Hogares (2009-2012). http://www.ennvih-mxfls.org/ennhiv-3.html 
Flisi, S., V. Goglio, E. .C Meroni, M. Rodrigues y E. Vera-Toscano. 2017.

Measuring occupational mismatch: Overeducation and overskill in Europe-Evidence from PIAAC, Social Indicators Research, 131(3), 12111249.

Freeman, R.B. 1976. The Overeducated American,.New York Academic Press. 1977. The decline in the economic rewards to college education, The Review of Economics and Statistics, 18-29.

Kucel, A. 2011. Literature survey of the incidence of over-education: A sociological approach, Revista Española de Investigaciones Sociológicas (REIS), 134(1): 125-142.

Leuven, E., y H. Oosterbeek. 2011. Overeducation and mismatch in the labor market, en E.A. Hanushek, S. Machin y Hoessmann (comps.), Handbook of the Economics of Education, vol. 4, Elsevier, pp. 283-326.

McGuinness, S. y K. Pouliakas. 2017. Deconstructing theories of overeducation in Europe: A wage decomposition approach, en S. W. Polachek et al. (comps.), Skill Mismatch in Labor Markets, Emerald Publishing Limited, pp. 81-127.

Mehta, A., J. Felipe, P. Quising y S. Camingue. 2011. Overeducation in developing economies: How can we test for it, and what does it mean? Economics of Education Review, 30(6): 1334-1347.

Mincer, Jacob. 1974. Schooling, Experience, and Earnings. New York: Columbia University Press. National Bureau of Economis.

Pissarides, C. A. 2000. Equilibrium unemployment theory. MIT press. 
Quinn, M.A. y S. Rubb. 2006). Mexico's labor market: The importance of education-occupation matching on wages and productivity in developing countries, Economics of Education Review, 25(2): 147-156.

Reis, M.C. 2017. Educational mismatch and labor earnings in Brazil, International Journal of Manpower, 38(2): 180-197.

Rumberger, R. W. (1981). Overeducation in the US labor market. Praeger Publishers.

Sattinger, M. 1993. Assignment models of the distribution of earnings, Journal of Economic Literature, 31(2): 831-880.

Sicherman, N. 1991. "Overeducation" in the labor market, Journal of Labor Economics, 9(2): 101-122.

----- y O. Galor. 1990. A theory of career mobility, Journal of Political Economy, 98(1): 169-192.

Thurow, L.C. 1975. Generating Inequality, Basic Books.

Verdugo, R. R., \& Verdugo, N. T. 1989. The impact of surplus schooling on earnings: Some additional findings. Journal of Human Resources, 629643. 